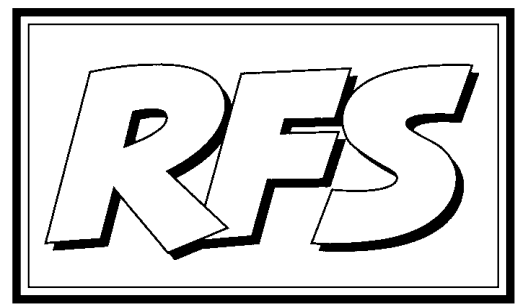

Revista de Fomento Social, 54 (1999), 221-244

\title{
Implicaciones de los sistemas de contabilidad de gestión sobre los recursos humanos
}

Los recursos humanos tienen una importancia creciente en todas las facetas de la gestión empresarial. A pesar de ser un recurso con un peso cada vez menor en la estructura de costes de las empresas, debido a una mayor automatización de los procesos y al desarrollo de la tecnología, se han convertido en un factor estratégico para la competitividad empresarial. La contabilidad, como responsable de ofrecer información relevante para la toma de decisiones, no puede permanecer ajena a esta realidad.

Por ello, frente a los sistemas contables que han venido implantando tradicionalmente las empresas, preocupados por la simple determinación del coste de personal, la gestión de los recursos humanos debe orientarse hacia la potenciación de valores y actitudes en los individuos.

En este artículo, su autora expone sus conclusiones acerca de las implicaciones que las modernas herramientas surgidas recientemente en el ámbito de la Contabilidad de Gestión tienen sobre los recursos humanos. Muchos de sus 
comentarios son fruto de los contactos mantenidos con empresas que han decidido implantar los denominados «sistemas de costes y de gestión basados en las actividades» y de la experiencia adquirida tras la participación en proyectos encaminados hacia el diseño de estos sistemas.

\section{Pilar TIRADO VALENCIA (*)}

\section{Introducción}

Durante varias décadas, la racionalización y el control del consumo de recursos en el desarrollo de la actividad empresarial y el análisis del proceso interno de creación de valor en las empresas han sido funciones de la disciplina conocidacomo Contabilidad de Costes.

La Contabilidad de Costes surge a finales del siglo pasado durante la Revolución Industrial, ante la necesidad de introducir en las valoraciones de los productos y en el control de gestión una nueva categoría de recursos que ganan importancia en la estructura de las empresas: los costes indirectos. Entre estos últimos destacan las amortizaciones derivadas de una mayor automatización, los costes administrativos originados por la mayor complejidad de las organizaciones y los costes comerciales, tras el desarrollo de las comunicaciones y el nacimiento de las primeras multinacionales.

Desde entonces, y hasta finales de la década de los ochenta, la Contabilidad de Costes ha permanecido fiel a los principios en los que se inspiró en sus orígenes, introduciendo tan sólo algunos mecanismos que reflejasen la evolución sufrida por ciertas clases de costes, como por ejemplo, la determinación y el análisis de los denominados costes fijos o independientes del volumen de produccióno, el cálculo de los llamados costes de subactividad o relacionados con la capacidad ociosa. Estas clasificaciones de los costes y la introducción de los costes estándares y del análisis presupuestario han dado lugar a los distintos sistemas contables que tienen implantados hoy la mayoría de las empresas.

En todos estos sistemas, conocidos como tradicionales, la variable coste y, en

(*)Profesora del Departamento de Gestión y Métodos Cuantitativos de la Facultad de C.E. y Empresariales (ETEA), adscrita a la Universidad de Córdoba. 
concreto, su cálculo y su control han dominado los informes internos, más preocupados por ofrecer información financiera de carácter cuantitativo que en reflejar aspectos cualitativos difícilmente cuantificables, como por ejemplo, el grado de satisfacción de los clientes, la motivación del personal, el nivel de compromiso con la calidad, la capacidad de adaptación a las ideas innovadoras, etc., aspectos todos ellos de vital importancia en la creación de valor añadido.

La preponderancia de la variable coste sobre estos otros aspectos estaba justificada en épocas pasadas en entornos en los que la mejor forma de competir en los mercados era ofreciendo los productos a los precios más bajos posibles, ya que éstos estaban aún poco diferenciados y las sucesivas crisis económicas hicieron que la reducción de los costes fuese el principal origen de ventajas competitivas.

En estas circunstancias, el coste es el factor que más incide en la competitividad de la empresa lo que justifica los enormes esfuerzos dedicados en las organizaciones a su cálculo y control, a la elaboración de los presupuestos y a la determinación de las desviaciones entre los datos reales y los presupuestados a priori.

Sin embargo, ante la globalización de los mercados y el incremento de la competencia, la Contabilidad de Costes se ha mostrado insuficiente para dar respuesta a las necesidades de información para la toma de decisiones. Las empresas deben ser excelentes y esto sólo se consigue ampliando el ámbito de actuación de la Contabilidad hacia otras variables claves de éxito comola calidad, el tiempo de entrega, la innovación, etc. Como resultado de esta ampliación surge la Contabilidad de Gestión.

La Contabilidad de Gestión es la disciplina que tiene como objetivo dar respuesta a las demandas de información de los responsables de la toma de decisiones de carácter operativo, es decir, relativas a la ejecución de los procesos internos de la organización. Entre los aspectos que la caracterizan se pueden destacar los siguientes:

- Amplía su ámbito de actuación hacia otras variables claves más allá del coste, ofreciendo tanto información cuantitativa como cualitativa, yaqueestaúltima ha estado ausente de los informes por estar basada en estimaciones y apreciaciones que a veces son subjetivas y porque la contabilidad ha estado preocupada por ofrecer datos verificables de forma objetiva.

- Extiende el análisis hacia el entorno. Esto supone incluir en los informes aspectos obviados hasta el momento, como por ejemplo, el estudio de la incidencia del comportamiento de los proveedores sobre la cadena de valor 
de la empresa, los efectos sobre la cadena de valor de los clientes e incluso el análisis de la posición relativa de la empresa con respecto a los competidores.

- Mantiene una visión global de la organización ya que las actuaciones individuales sólo fomentan resultados parciales o aislados que pueden ir en contra de los objetivos generales.

- Se centra tanto en el corto como en el largo plazo.

- Entre sus objetivos destaca la necesidad de orientar los procesos de mejora continua en las empresas, para llegar a ser excelentes en todos los sentidos.

- Refleja los cambios que se han producido en los sistemas de producción de las empresas, tecnológicamente más avanzados, más flexibles y precisos, con ciclos más cortos y capaces de adaptarse rápidamente a los cambios en las especificaciones de los clientes.

- Se centra en el análisis horizontal de la organización a través del estudio de los procesos empresariales, más que en las funciones basadas en una perspectiva vertical.

- Es consciente de la importancia que juegan los recursos humanos en la obtención de ventajas competitivas sostenibles. Uno de los aspectos que más ha incidido en estas últimas décadas en la revolución que están sufriendo las organizaciones es la constante preocupación por los recursos humanos que la integran. Las empresas son conscientes de que es necesario actuar sobre aquellas variables que están relacionadas con el comportamiento y la motivación del personal.

A continuación, se analizan algunos efectos perjudiciales de los sistemas tradicionales de costes sobre ciertos aspectos relacionados con la gestión de los recursos humanos, así como la evolución que en este terreno han experimentado los sistemas contables, y más concretamente, las implicaciones que sobre los recursos humanos tiene el diseño y la implantación de una de las últimas filosofías surgidas en el ámbito de la Contabilidad de Gestión: los sistemas de costes y de gestión basados en las actividades.

\section{Efectos de los sistemas de costes tradicionales sobre los recursos humanos}

Entre los principios inspiradores de la Contabilidad de Costes, una de las hipótesis que más ha influido en el diseño y funcionamiento de los sistemas contables actuales es la suposición de que existe un factor productivo dominante 
en la estructura de costes de las empresas. Este factor es sin duda el coste de personal.

La importancia concedida al registro exhaustivo de los consumos relacionados con la mano de obra, a su localización por centros o funciones y a su control por medio del cálculo de desviaciones estaba justificada en entornos poco automatizados, en los que ese tipo de costes tenía un peso específico considerable.

Esto explica la cantidad de tiempo y de recursos dedicados en las empresas a la determinación del coste de personal, a su registro, al cálculo de la productividad de la mano de obra, a la clasificación de esta última en directa e indirecta, y en general, a toda una serie de actuaciones encaminadas a controlar su coste y la cantidad de horas de mano de obra que se consumen en el desarrollo del proceso de producción.

La importancia concedida al coste de personal ha sido tal que este factor ha servido de base al denominado método de las secciones homogéneas, en el que se fundamentan la inmensa mayoría de los sistemas de costes implantados en la actualidad en las empresas. Se trata de un procedimiento que permite el reparto de los costes indirectos entre los diferentes productos o servicios. Este tipo de costes es más difícil de asociar a un objeto concreto ya que no se identifican en estos últimos de forma clara y evidente; entre ellos destacan la amortización de las construcciones, los equipos informáticos o el mobiliario, el material de oficina, el coste del personal que no está vinculado directamente a la fabricación o a la prestación del servicio sino que desarrolla actividades generales, etc.

El método de las secciones homogéneas imputa los consumos indirectos en dos fases: en la primera, estos costes se localizan en las diferentes funciones o centros de coste; en la segunda, el coste de los centros de coste se reparte entre los productos y servicios. En esta segunda etapa, se utilizan las horas de mano de obra y el coste por hora de mano de obra como principales, e incluso únicos, criterios de reparto, lo que explica la necesidad de llevar a cabo un control periódico de ambas magnitudes. En la literatura contable estos criterios de reparto se denominan unidades de obra.

Sin embargo, la aplicación de este método y la elaboración de presupuestos basados en costes estándares han venido desvirtuando sistemáticamente los costes de producción y han originado que las personas tomen decisiones poco eficientes.

Entre las implicaciones que tiene la utilización de las horas de mano de obra como unidad de obra se pueden destacar las siguientes: 
- La gestión se centra en el mero cálculo del coste de personal, cuando lo realmente importante es el potencial de los recursos humanos y no su coste.

- Si se utilizan las horas de mano de obra como criterio general de reparto de los costes, los productos intensivos en mano de obra son sobrevalorados ya que a pesar de que son los menos automatizados, reciben un mayor porcentaje de los costes de esta naturaleza, por lo que se obtienen valoraciones poco precisas y dan lugar a subsidios cruzados o intercambios de costes en las valoraciones de los productos.

- Al utilizar como criterio de reparto un parámetro basado en el volumen de producción, los subsidios se agravan aún más ya que se sobrecargan los productos que se fabrican en grandes cantidades frente a los productos y pedidos especiales, a pesar de que estos últimos requieren más costes de diseño, cambios de máquinas, tramitación de órdenes de pedido especiales, embalaje, etc. Por ejemplo, si en una empresa se fabrican dos productos que consumen las mismas horas de mano de obra, pero uno de ellos es una referencia bastante estandarizada que se produce en grandes lotes y el otro se fabrica según las especificaciones de un cliente concreto, un sistema tradicional los valoraría con el mismo coste de producción, cuando evidentemente el segundo ha requerido unos mayores esfuerzos y ha consumido más recursos.

Por otra parte, el llamado control por excepciones (1) o basado en el cálculo de desviaciones sobre los costes presupuestados, tiene una serie de efectos no deseados sobre el comportamiento de las personas, entre los que se pueden mencionarlos siguientes:

- Los estándares no motivan al personal para que consiga una mejora continua ya que los esfuerzos llegan hasta el mismo límite del estándar. Una vez que éste se ha alcanzado existe poca motivación para seguir mejorando.

- Los estándares frenan la creatividad de los recursos humanos, al dar por buenos los objetivos fijados en los presupuestos.

- La utilización de las desviaciones como herramienta para el control del personal y para la asignación de responsabilidades crea conflictos entre

(1)Para profundizaren el estudio de lallamada «Dirección por Excepción» puede consultarse: A. SÁez Torrecilla y Otros (1994), Contabilidad de Costes y Contabilidad de Gestión, capítulo 23, Madrid, Ed. McGraw-Hill, pp. 114-143. 
departamentos. Las personas pierden la visión global de la organización y se centran en la mejora de sus funciones aún a costa del resto. Por ejemplo, para reducir las desviaciones en el precio de las materias primas y otros aprovisionamientos, muchos jefes de compras han aprovechado al máximo los descuentos por volumen de pedido (rappels), adquiriendo grandes lotes aún cuando la mercancía no era necesaria, ocasionando graves problemas a la función de almacenaje.

- Asimismo, la utilización de las desviaciones como base de los sistemas de incentivos ha desmotivado al personal, ya que en muchas empresas se fijan estándares teóricos poco realistas o inalcanzables, lo que provoca frustración en los empleados.

- La definición de la productividad de la mano de obra como uno de los indicadores básicos en el sistema de control de gestión ha generado altos niveles de inventarios y exceso de existencias, lo que incrementa aún más los costes de mantenimiento de almacenes, localización de mercancías, transporte, obsolescencia, mermas y roturas, etc. y es el origen de un buen número de ineficiencias.

Todos estos efectos y sus implicaciones obedecen al profundo cambio que se ha producido en el modelo actual de empresa, en el que la mano de obra es un recurso cada vez más escaso por estar menos presente en términos cuantitativos, debido a que la revolución tecnológica sustituye personas por máquinas, pero a su vez, es más estratégico ya que el papel que juegan los recursos humanos es cada día más importante y se requiere un personal mucho más cualificado.

La evolución de la Contabilidad de Costes hacia una Contabilidad de Gestión así como la pérdida de peso específico del coste de personal en la estructura de costes de las empresas, han supuesto un cambio drástico en las competencias de esta última disciplina con respecto a los recursos humanos. Es necesario un cambio de orientación que permita adaptar la información contable al modelo de empresa actual en el que lo importante ya no es la determinación del coste de personal sino su correcta gestión; esta última implica la potenciación de una serie de valores y actitudes en las personas.

Para ser excelentes, lo importante no es minimizar los costes de los recursos humanos ni su control exhaustivo, sino que es necesario fomentar en el personal una serie de valores como la creatividad o el compromiso con la calidad, formarlos adecuadamente y confiar más en su propio autocontrol. 


\section{Los recursos humanos ante las nuevas tendencias en contabilidad de gestión}

La evolución hacia una Contabilidad de Gestión exige un cambio en los valores y comportamientos que deben potenciarse en las personas. Algunos de estos aspectos son compartidos por las numerosas herramientas surgidas recientemente dentro del ámbito de actuación de esta disciplina.

En general, el sistema contable no debe fomentar el logro de resultados de carácter financiero o las actuaciones dirigidas hacia la maximización de los beneficios a corto plazo, a través de la búsqueda de una mayor rentabilidad y productividad de los recursos humanos; por el contrario, las últimas corrientes propugnan un mayor énfasis en el desarrollo de una serie de actitudes que garanticen la subsistencia de la empresa a largo plazo.

A continuación se analizan algunos de los comportamientos y valores que debe favorecer un sistema de Contabilidad de Gestión en las personas y en el propio modelo de gestión, frente a las actitudes que han impulsado los sistemas de Contabilidad de Costes tradicionales.

\subsection{Contribución a la creación de valor añadidofrente a reducción de los costes a corto plazo}

Los sistemas contables tradicionales han fomentado la obtención de resultados inmediatos que ponen en peligro el crecimiento e incluso la subsistencia de la empresa a largo plazo. Las políticas de racionalización de costes abordadas de forma aislada mejoran temporalmente la rentabilidad de los productos, pero por otra parte, acaban empeorando la posición competitiva de la empresa si no van acompañadas de una política más global que incorpore también el estudio de los efectos que esta racionalización puede tener sobre otras variables. Por ejemplo, en muchas empresas, la obsesión por minimizar los costes ha supuesto la adopción de políticas de compras a proveedores al precio más bajo posible, aún a costa de sacrificar la calidad o la entrega a tiempo de los aprovisionamientos. Esta actuación es comprensible si se evalúa al personal tan sólo en términos de coste, algo que ha venido siendo frecuente cuando la asignación de responsabilidades se realiza a través de los centros de coste o de los centros de beneficios. Estos centros suelen ser divisiones funcionales de la organización, al frente de las cuales se coloca a una 
persona que responde de los costes en los que incurre el centro o de su contribución a la generación de beneficios.

La mejor forma de asegurar que los avances logrados se van a mantener en el futuro es evaluando la actuación de los empleados en términos de valor añadido y no exclusivamente en términos de racionalización de los costes. Esta preocupación ha desembocado en el nacimiento en el ámbito de la Contabilidad de Gestión de técnicas como el «Análisis del Valor Añadido» (Value Added Analysis), una de cuyas parcelas, el «Análisis del Valor Añadido por los Empleados» (Personal Value Added) estudia específicamente cómo colaboran los empleados en la creación de valor, e igualmente, cómo la organización colabora en la creación de valor o compromiso en su personal, por ejemplo, a través de programas de motivación y de incentivos.

\subsection{Mejora de la eficiencia frente a aumento de la productividad}

La productividad de la mano de obra ha sido uno de los indicadores de control utilizado con más frecuencia por las empresas para evaluar a sus empleados, ya que su incremento supone, supuestamente, un mejor aprovechamiento de la capacidad de los recursos humanos. Por otra parte, una mejora de la productividad disminuye indirectamente los costes de personal por unidad obtenida. Las economías de escala así logradas mejoran en apariencia la rentabilidad de los productos.

Sin embargo, la utilización de la productividad como indicador de control ha tenido efectos contraproducentes en la mayoría de las empresas. Esto es debido a que, para mejorar la productividad, los empleados intentan incrementar al máximo su rendimiento, lo que provoca:

- Un aumento innecesario de los inventarios que genera excesos de existencias en almacén.

- Problemas de gestión de la capacidad en otras funciones. Este efecto se analiza con más detenimiento en un apartado posterior.

- Rivalidad entre empleados, angustia, fatiga y falta de motivación que terminan por socavar la moral del personal.

- Efectos parciales, ya que la máxima productividad se consigue a veces a costa de otras variables, como por ejemplo, un deterioro de la calidad.

- Un riguroso sistema de supervisión y de inspecciones que detecte los errores 
provocados por un personal al que se evalúa en función de la cantidad de output que genera, pero no de la calidad de este output.

Por el contrario, en los actuales sistemas deContabilidad de Gestión las medidas de los sistemas de control están orientadas hacia la evaluación de la eficiencia en términos globales.

Los informes presentan un conjunto de indicadores que están interrelacionados entre sí y que incluyen, por ejemplo, el número de productos defectuosos, el tiempo de ciclo, la rentabilidad, los cambios introducidos en el diseño del producto, lacuota de mercado, el número de sugerencias procedentes de los empleados, el número de productos devueltos, el número de reclamaciones, el número de componentes comunes, etc.

En este sentido se orientan determinadas herramientas surgidas en el ámbito de la Contabilidad de Gestión como el «Control de la Calidad Total» (Total Quality Management) o los denominados sistemas «Justo a Tiempo» (Just in Time), que introducen otro tipo de mediciones.

En general, el sistema de medidas debe quedar integrado en lo que se conoce como «Cuadro de Mando Integral» (Balanced Scorecard), entre las que se encuentran una serie de indicadores relacionados con el personal, como por ejemplo, la capacidad de comunicación, el número de ideas o sugerencias que aporta, la valoración que merece entre sus compañeros, la utilización eficaz de los recursos de los que dispone, el grado de identificación con los objetivos generales de la organización, etc.

\subsection{Gestión del flujo frente a pleno rendimiento}

La idea de que el personal nunca debe permanecer ocioso está implícita en la utilización de la productividad como medida del desempeño de los empleados. Hasta hace pocos años, la infrautilización de la fuerza de trabajo suponía un despilfarro de recursos difícilmente justificable.

Sin embargo, las empresas han tratado el tema de la capacidad de forma fragmentada, como si se tratase de un asunto competencia de cada función de forma aislada. Esto supone obviar las interrelaciones que existen en el seno de la organización, lo que fomenta la búsqueda de óptimos locales debido al mantenimiento de una visión funcional y ha dado lugar a excesos de inventarios, una menor facturación y ha disparado los gastos, ya que no tiene en cuenta la existencia de 
restricciones o cuellos de botella en el sistema. Un cuello de botella es un recurso con capacidad limitada. Aumentar la capacidad del sistema por encima de la capacidad de los cuellos de botella es absurdo ya que se termina por saturar el proceso, incapaz de absorber los recursos que llegan al «embudo» que ocasiona el cuello de botella. Poreso, se debe pedir al personal que adecue su ritmo de trabajo al flujo marcado por los recursos que generan cuellos, para no colapsar el proceso de producción y mantener un equilibrio adecuado. Esto exige un mayor trabajo de coordinación entre los trabajadores de distintas funciones.

La denominada «Teoría de las Limitaciones» (Theory of Constraints) es la herramienta surgida en el ámbito de la Contabilidad de Gestión para tratar de dar respuesta a los problemas surgidos con respecto al uso óptimo de la capacidad.

La aplicación de esta filosofía de gestión de la capacidad sólo es posible si se cuenta con un personal debidamente formado, capaz de percibir cuáles son las necesidades del sistema. Además, es necesario un equipo humano polivalente que pueda atender las necesidades de personal de los cuellos de botella cuando sea necesario, o que se pueda reasignar a otras tareas cuando los recursos que no son cuellos tengan exceso de capacidad.

\subsection{Colectividad frente a individualismo}

Las organizaciones piramidales, con un gran número de niveles jerárquicos, se han mostrado poco operativas a la hora de tomar decisiones en las que la flexibilidad, la innovación y el tiempo de respuesta son variables claves para el éxito.

Por otra parte, frente a una estructura vertical basada en la división funcional de las empresas, es necesario mantener una visión horizontal de la organización en la que la toma de decisiones se realice teniendo en cuenta sus efectos a lo largo de los procesos empresariales que constituyen su cadena de valor. Al centrar la gestión sobre los procesos se trascienden las barreras funcionales y es posible conocer las interrelaciones en el seno de la organización.

Actuando sobre los procesos y no sobre las funciones, los efectos encadenados de las decisiones se hacen más visibles, manteniendo una concepción global de la gestión y no por departamentos estanco. En este sentido se orienta la herramienta conocida como «Reingeniería de Procesos»(Business Process 
Reeingeering).

Al hablar de globalidad, efectos encadenados e interrelaciones en la toma de decisiones, implícitamente se hace referencia a la necesidad de abandonar los comportamientos aislados y de conseguir que la empresa esté formada por un colectivo de personas que actúa conjuntamente para alcanzar unos objetivos comunes, frente a una serie de individuos que actúan independientemente para lograr sus propios intereses.

Para ello, es necesario valorar la capacidad de las personas para trabajar en equipo, su grado de compromiso con los objetivos globales de la organización, su identificación con las metas marcadas, su capacidad para comprender los efectos que su actuación tiene sobre el resto de la cadena de valor; con respecto al sistema de gestión, éste debe ser capaz de evaluar las implicaciones que tiene la actuación de un empleado de forma global o colectiva. Para ello es fundamental el conocimiento de los llamados eslabones en la toma de decisiones. Hablamos de la existencia de eslabones cuando la ejecución de una tarea incide en la forma como se ejecutan otras.

\subsection{Gestión del potencial de los recursos humanos frente a determinación del coste de personal}

Los sistemas de Contabilidad de Gestión deben aportar información relativa al potencial de los recursos humanos y no centrarse simplemente en el cálculo y el registro del coste de personal. Los recursos humanos deben ser evaluados en función de su grado de compromiso con los objetivos de la organización y de sus capacidades presentes y futuras. En esta evaluación deben primar una serie de valores en las personas, más que su coste o su rendimiento. Muchos de estos valores son difícilmente cuantificables y por eso no han sido introducidos hasta ahora en los informes contables.

Por otra parte, la determinación del coste de personal que realizan en la actualidad muchas empresas está desvirtuada, ya que se limita al coste de adquisición y compensación, obviando partidas tan importantes como las relativas a la formación, capacitación, sustitución, reclutamiento, selección, etc.

La gestión del potencial de los recursos humanos implica fomentar y favorecer en el personal ciertos aspectos entre los que debemos destacar la creatividad, flexibilidad, participación, aprendizaje, formación y capacitación, iniciativa, 
integración, motivación, compromiso (2), polivalencia y estabilidad. En general, la Contabilidad de Gestión debe valorar cualquier comportamiento que apoye el proceso de mejora continua en el que deben verse inmersas todas las empresas para garantizar su supervivencia. De este tipo de análisis surge el concepto de «Capital Intelectual» o conjunto de activos presentes y futuros relacionados con las personas que no siempre están reflejados en los estados contables y la necesidad de una «Gestión del Conocimiento» y no simplemente del coste.

Por otra parte, para asegurar que las decisiones se toman a tiempo deben abandonarse los comportamientos rígidos y burocráticos y dotar al sistema de la necesaria flexibilidad. Para ello es necesario fomentar el autocontrol y la toma de decisiones correctoras por parte de los empleados sin necesidad de autorización; esto sólo es posible gracias a una mayor formación del personal. Este aspecto se trata con mayor profundidad a continuación.

Finalmente, es necesaria una mejora en la comunicación entre los responsables de la gestión y los empleados. La apertura de canales de comunicación ágiles y fluidos es fundamental para fomentar las actitudes mencionadas anteriormente.

\subsection{Autocontrol frente a autoridad}

Los sistemas tradicionales no favorecen el autocontrol; por el contrario, los sistemas de control parecen estar justificados porque se desconfía de que los trabajadores puedan desempeñar por sí mismos sus tareas de la forma más eficiente. Esta idea explica la necesidad de controles precisos mediante ordenadores en la propia línea, muestreos periódicos para detectar las ineficiencias y la existencia de supervisores e inspectores. Con ello se ralentiza la toma de decisiones correctoras ante los problemas con los que se enfrentan los trabajadores, puesto que éstos podrían aportar soluciones de una forma más rápida y eficiente.

Frente a la existencia de un sistema de control exhaustivo, se debe concienciar e involucrar a los trabajadores: ellos deben ser los responsables de tomar sus propias decisiones, ya que son los que mejor conocen el trabajo que realizan. Para

(2) Para profundizar en la necesidad de un mayor compromiso de los recursos humanos con la organización y en la influencia del estilo de dirección sobre el nivel de compromiso, se puede consultar A. ARIZA MONTES (1998), «La influencia del Estilo Directivo en el Compromiso Organizativo», Revista de Fomento Social, n 53 , pp. 541-555. 
conseguirque los trabajadores sean auténticos colaboradores, surgen herramientas como el «Empowerment» $\mathrm{o}$ «Involvement»

Además, los indicadores de control relativos al comportamiento de los recursos humanos son medidas financieras y cuantitativas, relacionadas con el coste de personal, es decir, con la contraprestación que percibe el trabajador por la actividad desempeñada en la empresa, como por ejemplo, el coste por hora de mano de obra. Sería necesario introducir variables no financieras en las mediciones, tales como la satisfacción, el grado de identificación con la organización, el cumplimiento de los objetivos estratégicos, la capacidad de desarrollo del personal, etc., fundamentales en un proceso de mejora continua y en la obtención de ventajas competitivas sostenibles.

Es cierto que en muchos casos estas medidas no ofrecen nada más que una aproximación de la realidad, puesto que se trata de variables difícilmente medibles de forma precisa; para estimarlas, se podrían realizar cuestionarios periódicos en los que los empleados expusieran sus aspiraciones, su percepción sobre el ambiente de trabajo, sus inquietudes, etc.

En el cuadro $n^{\circ} 1$ se resumen los aspectos puestos de manifiesto en los apartados anteriores.

\section{Los sistemas de costes y de gestión basados en las actividades. Importancia de los recursos humanos}

Los sistemas de costes y de gestión basados en las actividades, denominados sistemas $\mathrm{ABC}$ y $\mathrm{ABM}$ respectivamente, surgen a principios de la década de los noventa ante la demanda de los responsables de la Contabilidad de Costes y del control de una herramienta que sistematizase toda la información necesaria para la toma de decisiones en el ámbito de la Contabilidad de Gestión.

Las metodologías ABC y ABM se basan en una hipótesis fundamental: son las actividades necesarias para desarrollar el proceso productivo las que consumen los recursos en la organización. Por lo tanto, la gestión empresarial se debe articular en torno a las actividades. La utilización de las actividades como eje central del sistema de información de las empresas responde a una evidencia: la eficiencia sólo se consigue mediante la gestión correcta de lo que se hace y, por tanto, a través del dominio y el control de las actividades y de su combinación en procesos.

La idea que está implícita en estos sistemas es que cualquier oportunidad de 


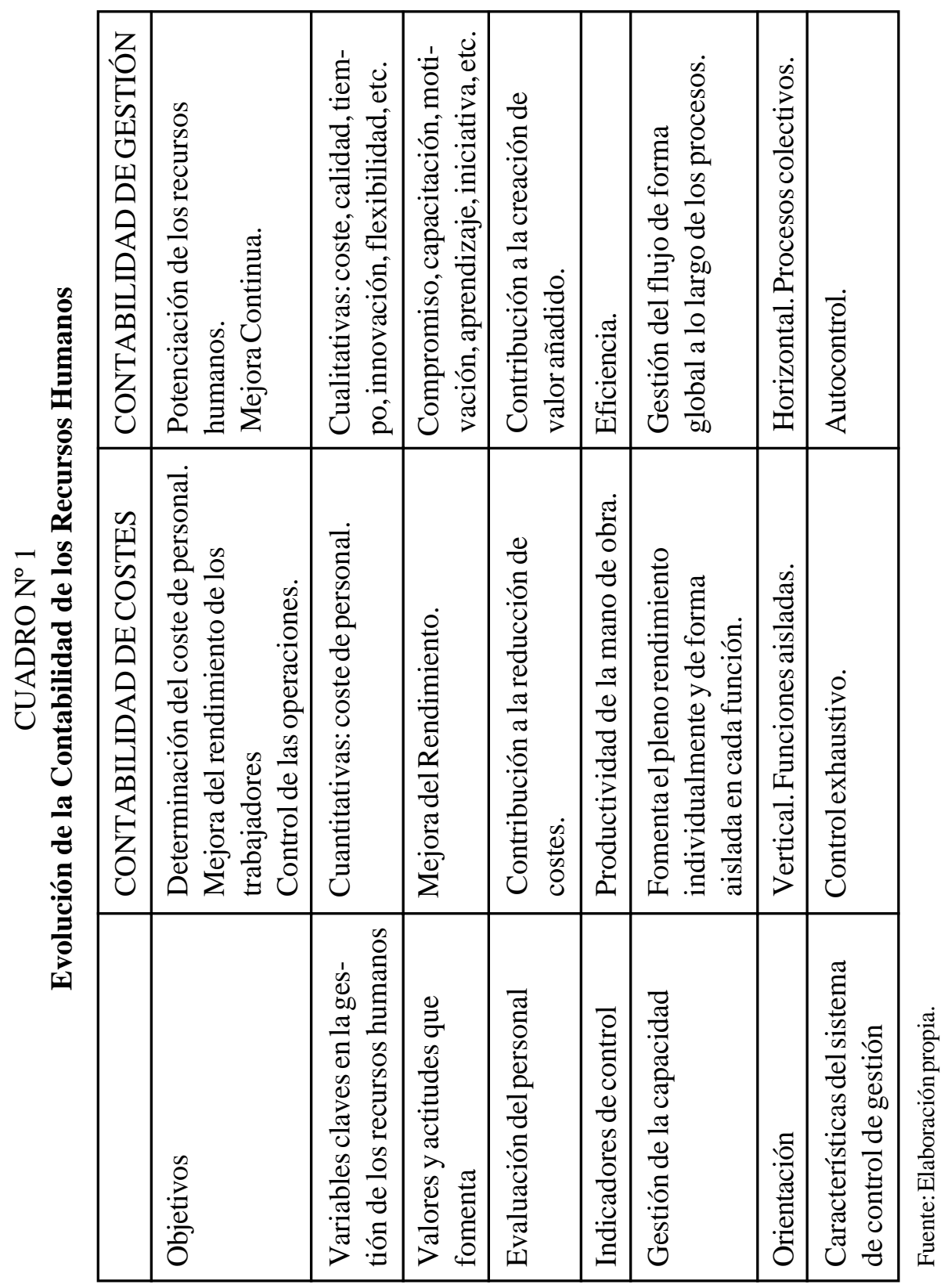


mejora se deriva de la posibilidad de racionalizar las actividades llevadas a cabo en el seno de la organización. Para ello, las actividades deben ser:

-identificadas,

- valoradas (sistemas ABC) y

- evaluadas (sistemas ABM).

El personal es una pieza clave en los sistemas $A B C$ y $A B M$ ya que su colaboración es imprescindible tanto en la fase de diseño como en la posterior implantación del modelo; es necesario contar en todo momento con la opinión y las sugerencias de los recursos humanos puesto que cada persona es quien mejor conoce el trabajo que realiza y, por tanto, quien mejor puede contribuir a la mejora continua de las actividades.

Una vez definidas las actividades, su valoración y la posterior asignación de su coste a los diferentes objetos (productos, servicios, clientes, etc.) se realizan utilizando parámetros distintos de las horas de mano de obra. Esta asignación se basa en criterios que miden el nivel de actividad ejecutado, como pueden ser: el número de componentes, el número de lotes, la cantidad de materiales consumidos, el número de órdenes de fabricación, los kilómetros recorridos, etc.

Con respecto a la evaluación de las actividades, ésta se realiza apoyándose en dos herramientas fundamentales:

- Los inductores de eficiencia o hechos causantes de las actividades. Se trata de identificar el porqué se ejecutan todas y cada una de las actividades. Una vez que se conoce su origen, es más fácil racionalizar su ejecución. Por ejemplo, la actividad movimiento de materiales carece de valor añadido, por lo que la empresa debe intentar eliminarla siempre que sea posible. Para ello, se debe buscar cuál es su origen; éste puede ser una deficiente distribución en planta, un sistema de información en los almacenes poco preciso o una mala formación de los trabajadores. La actividad sólo puede ser debidamente gestionada actuando sobre estas causas.

- Las medidas de la ejecución. Para cada actividad se deben definir una serie de indicadores que permitan controlar su ejecución. Estas medidas no sólo se expresan en términos de coste sino que también se refieren a otras variables claves; por ejemplo, el tiempo de ejecución de la actividad, el número de piezas defectuosas, etc.

\section{RFS}




\subsection{Apoyo de los sistemas ABC y ABM a la gestión del personal}

Los sistemas basados en las actividades pueden apoyar la gestión del personal en distintos sentidos:

A) Aspectos relacionados con la comunicación:

- Utilizan un lenguaje fácilmente comprensible por parte de todos los miembros de la organización: el de las actividades; en los sistemas ABC y ABM los informes se expresan en unos términos que pueden ser asimilados por todas las personas; esto fomenta la motivación y la creatividad ya que cualquier individuo puede aportar ideas sin que se requieran conocimientos financieros o contables específicos.

- Facilitan lafluidez en los canales de comunicación ya que la dirección y el resto del personal hablan un lenguaje común: los objetivos, los indicadores de control, los presupuestos, o cualquier oportunidad o problema surgido en el proceso, se expresan en forma de actividades, por lo que cualquier persona puede comprender qué es lo que se espera de ella. El sistema de información es más dinámico, facilitando el feedback y la participación.

- Permiten comprender mejor los efectos de una decisión. Las consecuencias económicas de las decisiones se hacen más visibles. Por ejemplo, ante una decisión de subcontratación, se pueden evaluar qué actividades dejarían de ejecutarse en la propia organización y qué recursos se podrían liberar de esta forma.

B) Aspectos relacionados con el compromiso:

- Favorecen el compromiso del personal. Los sistemas basados en las actividades exigen un alto grado de implicación por parte de los individuos, tanto en la fase de diseño como en su posterior implantación. En la definición de las actividades se requiere una estrecha colaboración del personal; sin su ayuda no es posible cubrir esta primera etapa del modelo, fundamental para su correcto funcionamiento. En muchas empresas, se comienza a diseñar el sistema con una serie de entrevistas con los trabajadores en las que éstos perciben desde el principio que son un elemento activo del sistema, lo que aumenta su motivación y su posterior colaboración en la generación de ideas. Las entrevistas presentan el inconveniente de que a veces las personas 
desvirtúan la información sobre las actividades ya que prefieren decir qué es lo que deberían hacer en lugar de lo que realmente hacen.

C) Aspectos relacionados con la participación:

- Facilitan la participación de los empleados en la toma de decisiones, ya que la mayor comprensión de las consecuencias de cualquier actuación así como la mejora de la comunicación inciden de forma positiva sobre ésta.

- Fomentan la generación de ideas en los procesos de mejora continua. La implicación del personal inherente a estos sistemas, además de motivar, es una extraordinaria fuente de sugerencias acerca de cómo mejorar y reducir las actividades que no generan valor añadido. La empresa aprovecha así una fuente potencial de ideas.

D) Aspectos relacionados con el desarrollo de sus tareas:

- Favorecen la colectividad y el trabajo en equipo ya en el modelo se deben identificar cuáles son los eslabones entre las actividades, es decir, los efectos inducidos de una actividad sobre otra. Cuando la ejecución de una actividad incide sobre otra, entre ellas existe un eslabón. De esta forma es posible que los trabajadores conozcan las interrelaciones con otras tareas.

- Facilitan el análisis del comportamiento de los agentes del entorno, en concreto de los proveedores, clientes y competidores, a través del estudio de las implicaciones que la ejecución de las actividades de la empresa tiene sobre éstos o de la comparación con la mejor forma de ejecutar esas actividades (Benchmarking). Mediante esta comparación se ofrece a los trabajadores una pauta de comportamiento en otras empresas o divisiones que han conseguido ser excelentes en la ejecución de una misma actividad. De esta forma, a los trabajadores les resulta más fácil comprender los efectos de sus actuaciones en el mercado y cómo están llevando a cabo los competidores las prácticas con las que consiguen ser más eficientes, si se expresan en términos de actividades.

- Facilitan la visión global de la organización. La utilización de los procesos como eje del sistema de control de gestión supone tener en cuenta las interrelaciones entre los distintos departamentos, puesto que un sólo proceso puede implicar varias funciones, abandonando la visión vertical de la organización estructurada por departamentos estanco sin relación aparente. 
Esto es posible gracias a que las actividades no se presentan en los sistemas $\mathrm{ABC}$ y $\mathrm{ABM}$ de forma aislada sino que deben ser enmarcadas dentro de los procesos horizontales de la organización. Para ello, se elabora lo que se conoce como mapa de actividades, que viene a ser como una especie de guía en la que cualquier actividad queda representada dentro de un proceso. Cualquier persona debe ser capaz de identificar las actividades que ejecuta dentro del mapa.

E) Aspectos relacionados con la administración del personal:

- Facilitan la previsión de las necesidades de trabajo y la planificación de las necesidades de cualificación y formación del personal mediante la previsión de las actividades que se vayan a acometer en el futuro. Los sistemas ABC y ABM pueden utilizarse para simular las necesidades de personal en el futuro, en función de las actividades que vayan a ser requeridas.

- Evalúan la eficiencia del personal y no sólo el coste ya que los sistemas de control basados en las actividades giran alrededor de las medidas de la ejecución; éstas sustituyen a los indicadores de control tradicionales y están referidas a actividades concretas y a variables como la calidad y el tiempo necesario para la ejecución de una tarea, que pueden ser la base de un sistema de incentivos.

F) Aspectos relacionados con el control de gestión:

- Cada individuo responde tan sólo de las actividades que ejecuta, sin tener en cuenta las variables que no son controlables por él, es decir, las que quedan al margen de las actividades que desarrolla. Además, la responsabilidad recae sobre la actividad y no sobre el coste. De esta forma, es más fácil imputar responsabilidades y diseñar un sistema de incentivos. A cada individuo se le valora por lo que hace, cómo lo hace y cómo influye en la ejecución del resto de las actividades (efectos inducidos o eslabones).

- Los empleados pueden ser responsables de su propia gestión y control; de esta forma, asumen la responsabilidad de adoptar las acciones correctoras oportunas ya que la planificación y la fijación de objetivos se expresan en términos de actividades. Esto significa que ellos mismos han de tomar sus propias decisiones y controlar sus procesos, detectando las desviaciones e ineficiencias. Esto se puede conseguir mediante la comunicación de los 
objetivos por medio de gráficos, ayudas visuales, diagramas de flujos, etc., que permiten a los trabajadores detectar los problemas, saber cuándo un proceso está fuera de control y cuáles son las actividades que deben ejecutar para corregir los errores, sin necesidad de consultar a un supervisor. En resumen, los sistemas $\mathrm{ABC}$ y $\mathrm{ABM}$ favorecen el control informal a través del autocontrol sobre las actividades que cada individuo ejecuta.

- El control de gestión recae sobre las causas de las desviaciones, no sobre los síntomas. El énfasis de los sistemas tradicionales en el cálculo de las desviaciones de los factores (materiales, mano de obra, etc.) pone de manifiesto como éstos centran el control sobre la utilización de los recursos. El objeto de estos sistemas son los inputs del proceso productivo y no los outputs (medidos en términos de calidad, tiempo, etc.). Por el contrario, los sistemas de control basados en las actividades conocen los hechos causantes de las desviaciones con respecto a los objetivos marcados (inductores), por lo que aseguran la toma de decisiones correctoras eficientes y facilitan la prevención de los efectos no deseados.

\subsection{Obstáculos en la gestión de los recursos humanos}

Tanto en la fase de diseño como en la implantación de los sistemas ABC y ABM se pueden presentar obstáculos relacionados con el comportamiento de los recursos humanos. En muchos casos, estos obstáculos no son exclusivos de estos sistemas, sino que se presentan en general cuando las empresas acometen cualquier iniciativa novedosa en el ámbito de la Contabilidad de Gestión, por miedo a lo desconocido.

- Resistencia de la organización al cambio. Una de las principales dificultades en el diseño e implantación de los sistemas de Contabilidad de Gestión es la tendencia del personal a mantener el statu quo; esta resistencia dificulta el desarrollo de filosofías innovadoras que suponen un cambio en la forma de concebir, no sólo el sistema contable, sino el negocio en general, lo que crea tensiones en los individuos, provoca recelos y puede poner en peligro el sistema. Una forma de combatir esta resistencia consiste en el desarrollo de un proyecto piloto que vaya generandolos primeros resultados rápidamente para que los individuos se vayan familiarizando con el modelo.

- Falta de apoyo por parte de la dirección. Si la dirección no está comprometida 
con el proyecto, éste está condenado a fracasar puesto que su diseño causa incomodidad en los responsables. La resistencia de los individuos sólo puede ser vencida si se cuenta con el apoyo de la dirección y si el personal es consciente de que ésta también está embarcada en el proyecto. En caso contrario, los sistemas $\mathrm{ABC}$ y ABM podrían ser vistos como un intento más de poner en práctica la filosofía de gestión «de moda», sin tomarse en serio los esfuerzos realizados y podrían ser considerados como algo pasajero.

- Desconfianza entre los empleados. Cuando se les pregunta a las personas acerca de su trabajo, suelen adoptar una actitud defensiva ya que al interrogar a la gente sobre lo que hace y a qué dedica su tiempo, se levantan muchas susceptibilidades que pueden hacer peligrar la implantación y los resultados del modelo. Este suele ser un obstáculo frecuenteque desvirtúa la información derivada del sistema, puesto que los empleados suelen ver este tipo de mejoras con cierto recelo y reaccionan contestando a los responsables del diseño del sistema lo que piensan que éstos quieren escuchar. La solución más adecuada ante esta situación es mejorar la comunicación, motivar a los trabajadores haciéndolos conscientes del papel que juegan en el diseño del sistema, mantenerlos continuamente informados de los avances del proyecto y asegurarles que sus puestos de trabajo no corren peligro. Algunas empresas han llegado incluso a publicar un folleto interno que se reparte periódicamente entre todos los empleados, en el que se muestran estos avances; en él se incluyen entrevistas a los responsables del diseño del sistema, información sobre los resultados obtenidos, etc.

- Gran volumen de información. La puesta en marcha de un sistema contable basado en las actividades requiere un conocimiento profundo de todos los aspectos de la organización: sus objetivos, estrategias, funcionamiento, etc. Esto se traduce en la necesidad de manejar un gran volumen de información, al menos inicialmente, que asegure que el modelo sea lo más completo posible. A veces, esto añade una mayor complejidad al sistema lo que hace que los individuos adopten una actitud defensiva y rechacen los primeros resultados. Para evitar este rechazo es imprescindible que los resultados se vayan ofreciendo escalonadamente ya que en términos de costes, en la mayoría de los casos es aplicable la regla de Paretto 80-20: el ochenta por ciento de los costes los ocasionan tan sólo un veinte por ciento de las actividades, que son las que requieren mayores esfuerzos y sobre las que nos debemos centrar en 
un principio para no sobresaturar los informes. Otro aspecto a tener en cuenta es la frecuencia con la que se van a elaborar los informes para que las personas no se sientan incómodas con el modelo ante un exceso de datos.

- Redefinición de los circuitos de información. El diseño de un sistema ABC y ABM supone una «ruptura» de los canales de comunicación tradicionales, lo que puede entrañar ciertas dificultades. En primer lugar, la información horizontal es más fluida al hacerse responsables los propios trabajadores de las actividades que ejecutan, desapareciendo buena parte de las tareas de supervisión. La información que reciben los trabajadores en los niveles jerárquicos más bajos gana peso. De esta forma, se intenta fomentar su creatividad y la generación deideas innovadoras tendentes a lograr una mejora continua.

Por otra parte, cambia la forma de obtener la información, y la frecuencia y el formato de los informes contables. Por ejemplo, desaparecen los detallados documentos sobre los centros de coste y sus desviaciones. Los responsables deben aprender a interpretar estos nuevos documentos lo que puede provocar ciertaresistencia.

\section{Conclusión}

Los sistemas de Contabilidad de Costes tradicionales han desvirtuado la información a la hora de tomar decisiones relacionadas con el personal y no han tenido los efectos deseados en el comportamiento de los individuos. La evolución de estadisciplinahaciauna Contabilidad de Gestión exige una mayor preocupación por la potenciación de los recursos humanos que por la determinación y la racionalización de su coste.

En esta nueva situación, es necesario que los individuos asuman una serie de valores que deben ser potenciados por el propio sistema contable, mediante el desarrollo de ciertas actitudes. Esto exige una reorientación en el tipo de información relativa a los recursos humanos que debe ser elaborada y de los indicadores que deben servir de base a los sistemas de control y de incentivos.

En este último sentido nacen los sistemas de costes y de gestión basados en las actividades ABC y ABM respectivamente. Ambos se basan en la idea de que las actividades deben ser el eje central del sistema contable. Cualquier intento de mejora debe expresarse en términos de actividades lo que facilita la comunicación 
y motiva a los trabajadores. La experiencia de las empresas que ya han implantado estos modelos ha demostrado que pueden facilitar la gestión de los recursos humanos, a pesar de los obstáculos que se pueden presentar al implantar cualquier nueva herramienta de gestión por miedo a lo desconocido.

Sin embargo, estos sistemas no son la panacea que solventa las enormes dificultades que entraña el tratar de gestionar un recurso de carácter humano ya que intervienen muchos factores emocionales que no están previstos por el sistema contable; tampoco ofrecen una «receta mágica» que resuelva todos los problemas relacionados con la gestión del personal ya que en muchos casos la «bondad» de estos modelos está condicionada por la cultura empresarial, por el estilo de dirección y por la disposición de los individuos a colaborar. Además, tan sólo empresas con un departamento de personal consolidado y con una dotación de recursos suficientes pueden afrontar el cambio de mentalidad y el volumen de información necesario para acometer un proyecto $\mathrm{ABC}-\mathrm{ABM}$. Este tipo de empresas suelen ser organizaciones de gran tamaño.

\section{Bibliografía}

ÁlvarezLópez, José y Blanco Ibarra, Felipe (1994), «Apoyo de la Contabilidad Directiva a la Motivación de los Recursos Humanos», Técnica Contable, $\mathrm{n}^{\circ}$ 547, Julio, pp. 449-462.

Bailes, Jack C. y Kleinsorge, Ilene K. (1992), «Cutting Waste with JIT», Management Accounting, Mayo, pp. 28-32.

Berry, William (1994), «The Human Resource Information System», Management Accounting, Enero, pp. 56-57.

Brimson, James A. (1995), Contabilidad por Actividades, Barcelona, Ed. Marcombo, 256 págs.

Gubman, Edward L. (1995), «El Protagonismo Creciente de los Recursos Humanos», Harvard Deusto Review, n 66, Mayo-Junio, pp. 43-51.

Johnson, H. Thomas y Kaplan, Robert S. (1988), La Contabilidad de Costes: Auge y Caída de la Contabilidad de Gestión, Barcelona, Ed. Plaza \& Janés, 248 págs.

Kaplan, Robert S. y Cooper, Robin (1999), Coste y Efecto. Cómo usar el ABC, el $A B M$ y el $A B B$ para mejorar la gestión, los procesos y la rentabilidad, 
Barcelona, Ed. Gestión 2000, 340 págs.

Kaplan, Robert S. y Norton, David P. (1996), Cuadro de Mando Integral, Barcelona, Ed. Gestión 2000, 321 págs.

Lorino, PhilipPe (1993), El Control de Gestión Estratégico. La Gestión por Actividades, Barcelona, Ed. Marcombo, 194 págs.

Player, R. Steven y Keys, David E. (1995), «Lessons from de ABM Batltefield: moving from pilot to mainstream», Journal of Cost Management, fall, pp. 3141.

Thorne, Helen y Gurd, Bruce (1995), «Some Human Aspects of Implementing Activity-Based Management», Journal of Cost Management, fall, pp. 50-57. Turney, Peter B.B. (1991), Common Cents, Hillsboro, Ed. Cost Technology, 322 págs.

—(1993), «Beyond TQM with Workforce Activity-Based Management», Management Accounting, Septiembre, pp. 28-31.

Veciana, Joser M ${ }^{\text {a }}$ y GenesCA, EnRic (1994), «Management y Competitividad en la Empresa Española», Alta Dirección, nº 174, Marzo, pp. 83-98. 